\title{
A Novel Low-Loss Diamond-Core Porous Fiber for Polarization Maintaining Terahertz Transmission
}

Islam, Raonaqul; Habib, Selim; Hasanuzzaman, G. K. M.; Rana, Sohel; Sadath, Md Anwar; Markos, Christos

Published in:

I E E E Photonics Technology Letters

Link to article, DOI:

10.1109/LPT.2016.2550205

Publication date:

2016

Document Version

Peer reviewed version

Link back to DTU Orbit

Citation (APA):

Islam, R., Habib, S., Hasanuzzaman, G. K. M., Rana, S., Sadath, M. A., \& Markos, C. (2016). A Novel Low-Loss Diamond-Core Porous Fiber for Polarization Maintaining Terahertz Transmission. I E E E Photonics Technology Letters, 28(14), 1537-1540. https://doi.org/10.1109/LPT.2016.2550205

\section{General rights}

Copyright and moral rights for the publications made accessible in the public portal are retained by the authors and/or other copyright owners and it is a condition of accessing publications that users recognise and abide by the legal requirements associated with these rights.

- Users may download and print one copy of any publication from the public portal for the purpose of private study or research.

- You may not further distribute the material or use it for any profit-making activity or commercial gain

- You may freely distribute the URL identifying the publication in the public portal 


\title{
A Novel Low Loss Diamond-Core Porous Fiber for Polarization Maintaining Terahertz Transmission
}

\author{
Raonaqul Islam, Md. Selim Habib, G. K. M. Hasanuzzaman, Sohel Rana, Md. Anwar Sadath, and \\ Christos Markos
}

\begin{abstract}
We report on the numerical design optimization of a new kind of relatively simple porous-core photonic crystal fiber (PCF) for terahertz (THz) waveguiding. A novel twist is introduced in the regular hexagonal PCF by including a diamond shaped porous-core inside the hexagonal cladding. The numerical results obtained from an efficient finite element method (FEM) which confirms a high-birefringence of the order $10^{-2}$ and low effective material loss of $0.07 \mathrm{~cm}-1$ at $0.7 \mathrm{THz}$ operating frequency. The proposed PCF is anticipated to be useful in polarization sensitive THz appliances.
\end{abstract}

Index Terms-Birefringence; porous-core; photonic crystal fiber; terahertz wave guidance

\section{INTRODUCTION}

$\mathrm{P}$ HOTONIC crystal fibers (PCFs) are often considered as waveguiding solutions to the well-known terahertz $(\mathrm{THz})$ technology. Since regular dielectrics absorb excessive power in this radiation band and dry air is transparent for $\mathrm{THz}$ waves, PCFs are so designed based on polymers that majority power fraction is guided in air-holes. Convenient porous fibers with air-cladding [1] and porous fibers with holey cladding [2],[3] have been reported earlier, where most of the power was propagated in low-loss air.

Meanwhile, significant applications such as $\mathrm{THz}$ time domain spectroscopy [4], [5] require polarization maintenance in the $\mathrm{THz}$ regime. Symmetry of a PCF is deliberately defected to induce high modal birefringence for $\mathrm{THz}$ polarization maintaining applications [6]-[10]. For example, Cho et al. demonstrated a plastic PCF [6] that exhibits a birefringence as high as $2.1 \times 10^{-2}$. However, the high propagation loss $400 \mathrm{~dB} / \mathrm{m}$ at $1 \mathrm{THz}$ makes the waveguide impractical for low-loss $\mathrm{THz}$ guiding. Use of rectangular airholes [7], [8] or elliptical air-holes [9], [10] in the core can achieve high-birefringence in the order $10^{-2}$. Nevertheless, the complicated rectangular or elliptical shapes introduce difficulty in practical realization of these structures and transmission loss is yet high due to absorption in the solid material. Moreover, these structures [7]-[10] use solid aircladding that involves direct connection with the outer environment where uncertain atmospheric losses may occur.

In this letter, we propose a simple and novel diamond-core PCF that shows simultaneously a high-birefringence of $\sim 10^{-2}$ and a low effective absorption loss of $\sim 0.07 \mathrm{~cm}^{-1}$ for both the polarization modes. Most importantly, it does not include any complex shaping and only circular air-holes are considered for the structure. To the best of our knowledge, this type of PCF has never been conferred in the $\mathrm{THz}$ research before.

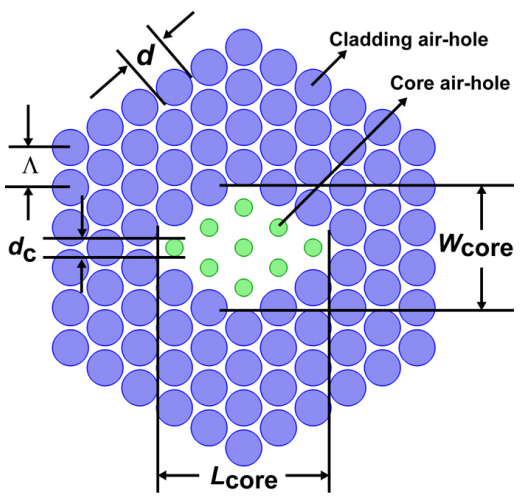

Fig. 1. Schematic diagram of the proposed diamond-core fiber structure.

\section{Design Methodology}

Fig. 1 shows the cross-section of the diamond-core structure. The core consists of diamond shape of air-hole pattern (green circles) whereas the cladding consists of regular hexagonal air-hole pattern (blue circles). The diameter of the core air-holes $\left(d_{\mathrm{c}}\right)$ was reduced compared to the diameter of the cladding air-holes $(d)$ in order to create the differential index contrast between the core and the cladding. Meanwhile, the principle design parameter chosen for this design is the length of the diamond-core $\left(L_{\text {core }}\right)$ because it determines the fiber dimension. The width of the core $\left(W_{\text {core }}\right)$ changes according to the $L_{\text {core. }}$ The pitch $(\Lambda)$, in other words, the distance between two adjacent air-holes of the same ring and two different rings throughout the structure, is related to $L_{\text {core }}$ as $L_{\text {core }}=6 \Lambda \cos 30^{\circ}-d$. That means we set the value of $L_{\text {core }}$ to find out the pitch of the fiber. The air-filling ratio $(d / \Lambda)$ of the cladding is kept fixed at 0.95 throughout the analysis. We have defined the core porosity as the ratio between core airhole areas to the total core area.

The background material considered for this design is cyclic olefin copolymer (COC), with a trade name of TOPAS. This polymer is preferred due to some of its excellent merits over other polymers such as PMMA or Teflon. For example, its refractive index is constant between $0.1-2 \mathrm{THz}$ [11], its bulk material loss is very low compared to other polymers $(\sim 0.2$ $\mathrm{cm}^{-1}$ at $1 \mathrm{THz}$ ) [11], it is humidity insensitive [12], and good for biosensing [13]. An efficient finite element method (FEM) based commercially available state-of-the-art COMSOL has been used to compute the modal properties of the proposed PCF. In addition, a perfectly matched layer (PML) is applied outside the computational domain to model the loss properties. The overall computational domain was $525 \mu \mathrm{m}$ when length of the diamond-core, $L_{\text {core }}$ was $400 \mu \mathrm{m}$. We used 21382 triangular vector edge elements and 3086 PML boundary 
elements to represent the structure, whereas the average element quality of the fiber was $\sim 0.9404$ which confirms the computational error less than $0.1 \%$.

\section{RESUlts AND Discussion}

First, we demonstrate the birefringence of the proposed fiber. Birefringence is basically the absolute difference between the refractive indices of $x$ and $y$ polarization modes expressed as [10]

$$
B=\left|n_{\mathrm{x}}-n_{\mathrm{y}}\right| \text {, }
$$

where $B$ stands for birefringence, $n_{\mathrm{x}}$ and $n_{\mathrm{y}}$ indicate effective refractive indices of $x$ and $y$ polarization modes respectively. Fig. 2 shows the calculated birefringence as a function of the core length at $20 \%, 40 \%$ and $60 \%$ core porosities. Apparently, birefringence is reduced when porosity is increased, whereas the variation of birefringence over core length is increased when the porosity is reduced. This happens because increment of porosity reduces the differential index contrast between the core and the cladding, resulting in less power inside the porous-core. As a consequence, the deliberate stress on the guided mode introduced by the diamond-core is decreased and thus birefringence is reduced. The birefringence for $20 \%$ porosity within $300-450 \mu \mathrm{m}$ and for $40 \%$ porosity within 300 $325 \mu \mathrm{m}$ at $1 \mathrm{THz}$ are high $\left(>10^{-2}\right)$, which are comparable to the previously published works [6]-[10]. The proposed fiber shows high-birefringence despite being exclusive of elliptical or rectangular shapes, which gives a tremendous advantage from fabrication point of view. The diamond-core structure can be realized more clearly from Fig. 3(a) in which the birefringence is illustrated as a function of frequency. It can be observed from Fig. 3(a) that for $40 \%$ porosity, the proposed design shows high-birefringence $\left(>10^{-2}\right)$ within a wide frequency band $(0.48-0.82 \mathrm{THz})$. To be specific, at $0.7 \mathrm{THz}$ and $40 \%$ porosity, the birefringence is maximum $(0.0105)$ for this design. The mode field distribution is shown in Figs. 3(b) and 3(c) where mode field is well confined inside the core.

We now discuss the most important loss in the $\mathrm{THz}$ regime which is effective absorption loss, expressed as [3]

$$
\alpha_{\mathrm{eff}}=\sqrt{\frac{\varepsilon_{0}}{\mu_{0}}}\left(\frac{\int_{\mathrm{mat}} n_{\text {mat }}|E|^{2} \alpha_{\text {mat }} \mathrm{dA}}{\left|\int_{\text {all }} S_{\mathrm{z}} \mathrm{dA}\right|}\right),
$$

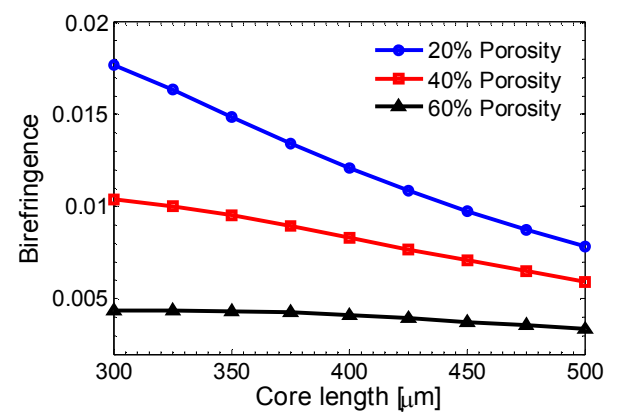

Fig. 2. Birefringence versus core length for different porosities at $1 \mathrm{THz}$.

where $\varepsilon_{\mathrm{o}}$ and $\mu_{\mathrm{o}}$ are the permittivity and permeability of vacuum, $n_{\text {mat }}$ is the refractive index of the material used, $E$ is the modal electric field, $\alpha_{\text {mat }}$ is the bulk material absorption loss and $S_{\mathrm{z}}$ is the $z$-component of the Poynting vector, $S_{\mathrm{z}}=$
$\frac{1}{2}(\vec{E} \times \vec{H}) \cdot \hat{z}$. The effective absorption loss as a function of core length of the proposed design is plotted in Fig. 4, where it is observed that the two polarization modes show different absorption loss and the loss is increased when porosity is reduced. The amount of absorbed power also increases with the frequency as shown in Fig. 5(a). This demonstration is performed after considering the incremental bulk-material loss of TOPAS with respect to the frequency as described in Ref. [7]. Although $60 \%$ porosity shows lower absorption loss than $40 \%$ (Fig. 5(a)), the porosity dependent loss is very low in the high-birefringence frequency band of interest 0.48-0.82 THz. Please note that the loss is $0.07 \mathrm{~cm}^{-1}$ for $y$-polarized mode and $0.076 \mathrm{~cm}^{-1}$ for $x$-polarized mode at $40 \%$ porosity and $0.7 \mathrm{THz}$, which is the clinical evidence of low polarization dependent loss. This matter can be understood more clearly from Figs. 5(b) and 5(c), which show that most of the power is propagated in air-holes for both the polarization modes. It is worthwhile to mention that the loss obtained at $40 \%$ porosity and $0.7 \mathrm{THz}$ is lower than the earlier reported ones [2]-[3], [6]-[10]. Next, we analyze the confinement loss of the fiber which is expressed as [14]

$$
\alpha_{\mathrm{CL}}=8.686 k_{0} \operatorname{Im}\left(n_{\mathrm{eff}}\right) \text {, }
$$

where $k_{0}=2 \pi / \lambda$ and $\operatorname{Im}\left(n_{\mathrm{eff}}\right)$ is the imaginary part of the refractive index, $n_{\text {eff. }}$ Fig. 6 shows the confinement loss property of the proposed fiber with respect to frequency. We observe that confinement loss is increased when frequency is decreased to the band of interest $(0.48-0.82 \mathrm{THz})$. Most importantly, the polarization dependent loss is low for all porosities (Fig. 6), which is of utmost importance for highbirefringence polarization maintenance.

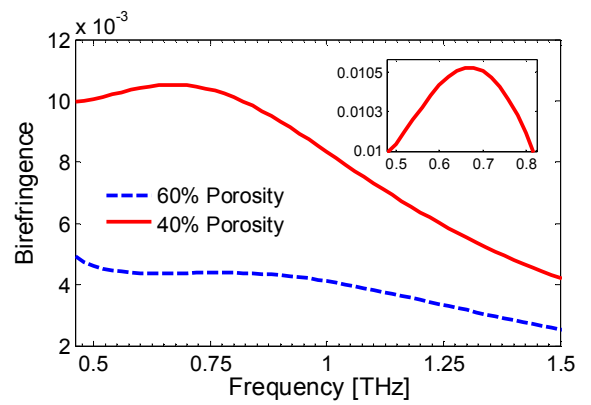

(a)

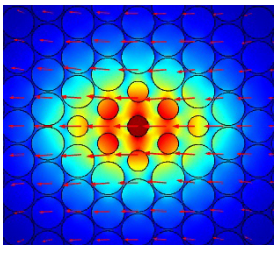

(b)

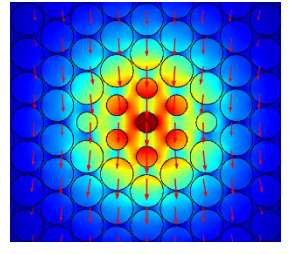

(c)
Fig. 3. a) Birefringence versus frequency, b) electric field of the mode power distribution for $x$-polarization mode and c) for $y$-polarization mode at $40 \%$ porosity, $L_{\text {core }}=400 \mu \mathrm{m}$ and $0.7 \mathrm{THz}$. 


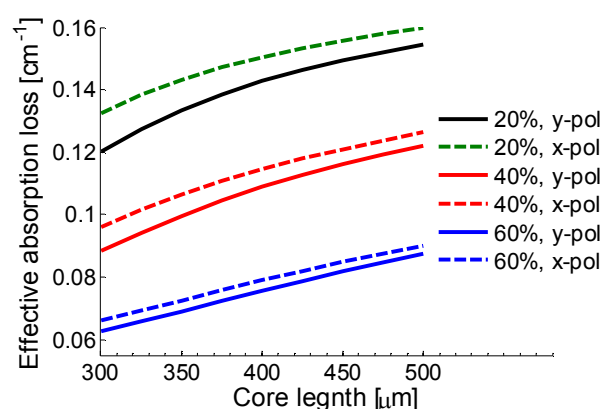

Fig. 4. Effective material absorption loss versus core length at $1 \mathrm{THz}$.

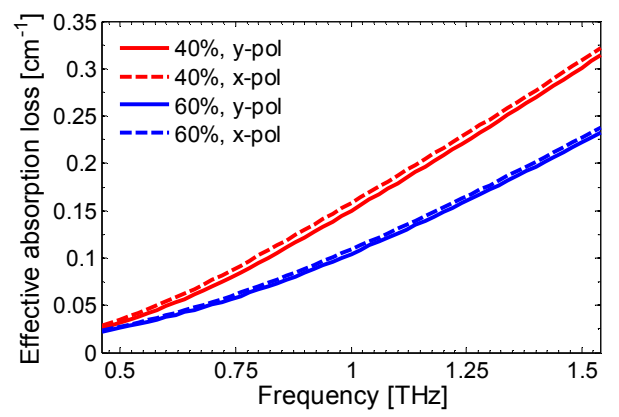

(a)

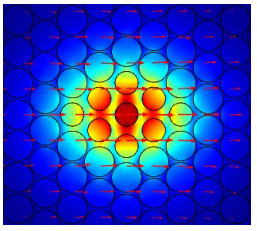

(b)

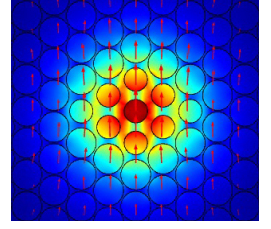

(c)
Fig. 5. a) Effective absorption loss versus frequency, b) electric field distribution of mode power flow at $L_{\text {core }}=400 \mu \mathrm{m}, 60 \%$ porosity and $1 \mathrm{THz}$ for $x$-polarization mode and c) for $y$-polarization mode.

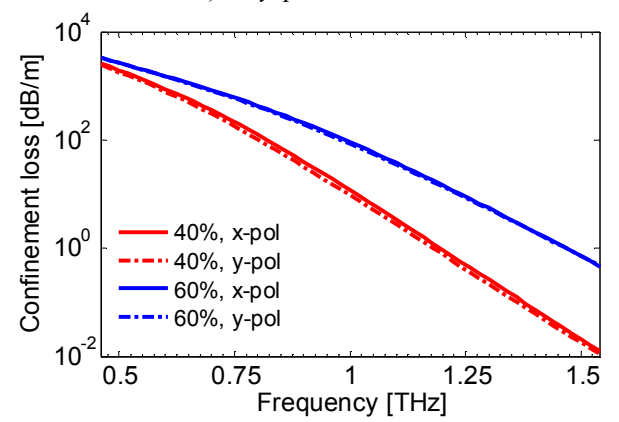

Fig. 6. Confinement loss versus core length for different porosities at $1 \mathrm{THz}$.

To calculate the bending loss, first we replace the bent fiber with its equivalent straight fiber. Then the effective refractive index of that fiber is found from the conformal transformation method which is used to calculate the leakage loss. The equivalent refractive index is given by [15]

$$
n_{\mathrm{eq}}(x, y)=n(x, y) \exp (1+x / R)
$$

where, $n(x, y)$ is the original refractive index profile of the fiber, $R$ is the bending radius and $x$ is the distance from the

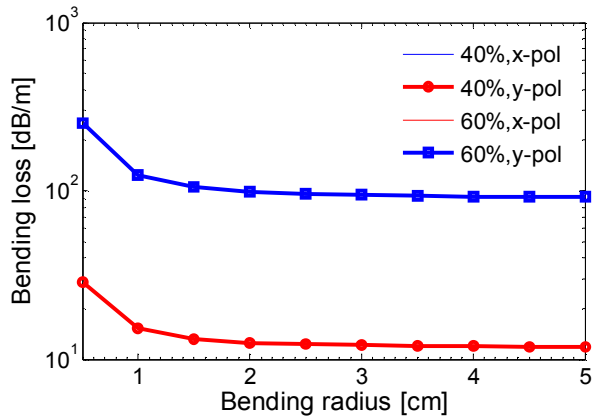

Fig. 7. Bending loss versus bending radius for different porosities and different polarization modes. Note that the bending loss for both polarization modes overlap each other.

center of the fiber. Fig. 7 shows the bending loss of the diamond-core PCF at various bending radii. We observe that the loss is reduced when either the bending radius is increased or the porosity is decreased.

Effective mode area of PCFs can be calculated by using [3]

$$
A_{\text {eff }}=\frac{\left[\int I(r) r \mathrm{dr}\right]^{2}}{\left[\int I^{2}(r) \mathrm{dr}\right]^{2}}
$$

where $I(r)=\left|E_{\mathrm{t}}\right|^{2}$ is the transverse electric field intensity distribution in the fiber cross section. Effective area of the proposed fiber is shown in Fig. 8, where it is observed that $A_{\text {eff }}$ is reduced with frequency and $y$-polarization modes show larger $A_{\text {eff }}$ than x-polarization modes. It is also observed that the effective area is increased with porosity. The reason is that when porosity is increased, the differential index contrast between the core and the cladding is reduced, which consequently increases the amount of power spreading outside the core. Note that $40 \%$ of core porosity shows a highbirefringence with a larger mode area.

At this point, we discuss the dispersion characteristics of the proposed fiber. Material dispersion of TOPAS is ignored due to its constant refractive index and only the waveguide dispersion is calculated using dispersion parameter [16]

$$
\beta_{2}=\frac{2}{c} \frac{\mathrm{d} n_{\mathrm{eff}}}{\mathrm{d} \omega}+\frac{\omega}{c} \frac{\mathrm{d}^{2} n_{\mathrm{eff}}}{\mathrm{d} \omega^{2}}
$$

where $\omega=2 \pi f$ and $c$ is the velocity of light in vacuum. In the high-birefringence frequency regime of the diamond-core PCF (0.48-0.82), it shows high dispersion (Fig. 7). The dispersion within $0.48-0.82 \mathrm{THz}$ at $40 \%$ porosity was $2.92 \pm 0.55$ $\mathrm{ps} / \mathrm{THz} / \mathrm{cm}$. However, polarization dependency of the dispersion parameter is very low as observed from Fig. 7, which is convenient for highly birefringent $\mathrm{THz}$ transmission.

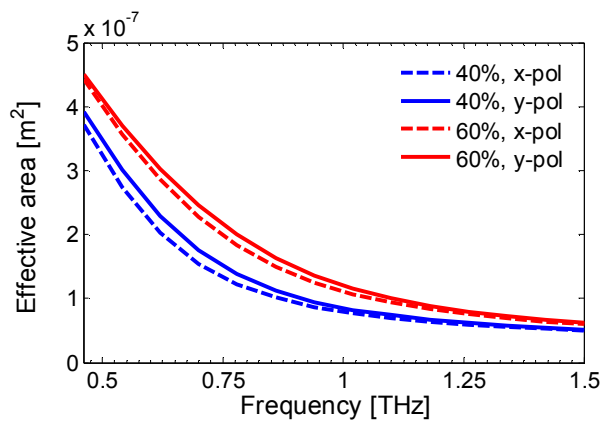

Fig. 8. Effective area as a function of frequency for different. 


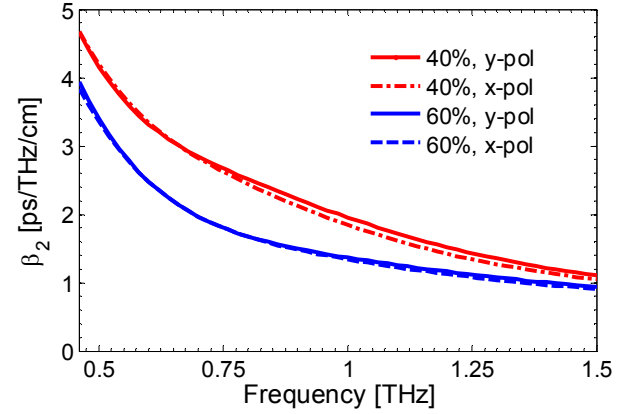

Fig. 9. Dispersion of the proposed PCF as a function of frequency at $L_{\text {core }}=$ $400 \mu \mathrm{m}$ and different porosities.

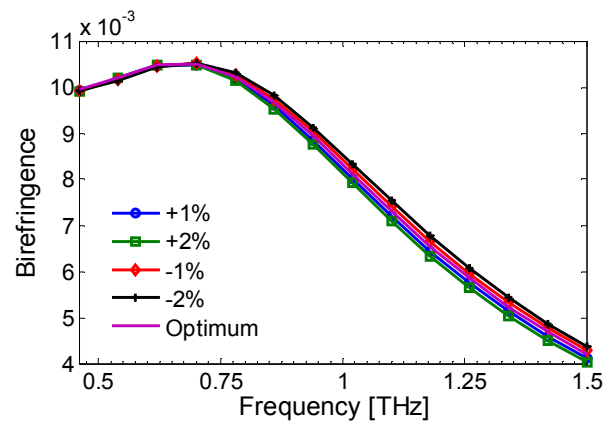

Fig. 10. Birefringence properties of the proposed fiber: optimum birefringence and effect of changing $L_{\text {core }}$.

We have also examined the diamond-core fiber from the fabrication point of view. It is well known that during a standard fabrication process, $\pm 2 \%$ variation may occur in the global structural parameter [17]. Owing that, we calculated the birefringence of the proposed fiber for up to $\pm 2 \%$ variation of $L_{\text {core }}$ from $400 \mu \mathrm{m}$ as shown in Fig. 10. It can be observed from Fig. 10 that the variations in $L_{\text {core }}$ do not affect the birefringence in the high-birefringence frequency band. We also explore the possibility of practical realization of the diamond-core PCF. In 2004, Barton et al. demonstrated the fabrication of microstructured polymer optical fibers (MPOFs) [18]. The method described in Ref. [18] used a combination of drilling and drawing of preforms into canes. The porous-core honeycomb bandgap fiber described in Ref. [19] has also been fabricated. Usually, air-hole patterns are drilled into the preform using a numerically controlled drill that guarantees smoother finishing and minimization of surface roughness. Therefore, this method should be sufficient for the fabrication of the proposed diamond-core structure, which would unleash the fact whether the fiber behaves similar to its numerical performance.

\section{CONCLUSION}

We have numerically investigated a novel PCF with highbirefringence in the $\mathrm{THz}$ region. At optimal design parameters, the proposed fiber exhibits a birefringence $>10^{-2}$ within $0.48-0.82 \mathrm{THz}$ and effective absorption loss of $\sim 0.07$ $\mathrm{cm}^{-1}$ for both the polarization modes. The three most significant properties of the diamond-core structure are its extraordinary simplicity, high-birefringence for a wide band $(\sim 0.34 \mathrm{THz})$ and near-zero polarization dependent loss. Thereby with proper utilization, the porous-core PCF would be a noteworthy contribution to the polarization maintaining THz technology.

\section{REFERENCES}

[1] A. Hassani, A. Dupuis, and M. Skorobogatiy, "Porous polymer fibers for low-loss terahertz guiding," Opt. Exp., vol. 16, no. 9, pp. 6340-6351, Apr. 2008.

[2] M. Uthman, B. M. A. Rahman, N. Kejalakshmy, A. Agarwal and K. T. V. Grattan, "Design and characterization of low-loss porous-core photonic crystal fiber," IEEE photonics J.,vol. 4, no. 6, Dec. 2012.

[3] S. F. Kaijage, Z. Ouyang and X. Jin, "Porous core photonic crystal fiber for low loss terahertz wave guiding," IEEE Photonics Technol. Lett., vol. 25, no. 15, Aug. 2013.

[4] M. B. Byrne, M. U. Shaukat, J. E. Cunningham, E. H. Linfield, and A. G. Davies, "Simultaneous measurement of orthogonal components of polarization in a free-space propagating terahertz signal using electrooptic detection," Appl. Phys. Lett., vol. 98, no. 15, pp. 15110

[5] N. Karpowicz, J. Dai, X. Lu, Y. Chen, M. Yamaguchi, H. Zhao, X.-C. Zhang, L. Zhang, C. Zhang, M. Price-Gallagher, C. Flectcher, O. Mamer, A. Lesimple, and J. Keith, "Coherent heterodyne time-domain spectrometrycovering the entire "terahertz gap"," Appl. Phys. Lett. vol. 92, no. 1, pp. 011131, 2008

[6] M. Cho, J. Kim, H. Park, Y. Han, K. Moon, E. Jung, and H. Han, "Highly birefringent terahertz polarization maintaining plastic photonic crystal fibers," Opt. Express, vol. 16, no. 1, pp. 7-12, 2008.

[7] S. Atakaramians, S. Afshar Vahid, B. M. Fischer, D. Abbott, and T. M. Monro, "Low loss, low dispersion and highly birefringent terahertz porous fibers," Opt. Commun., vol. 282, no. 1, pp. 36-38, 2009.

[8] R. Islam, M. S. Habib, G. K. M. Hasanuzzaman, R. Ahmad, S. Rana, and S. F. Kaijage, "Extremely high-birefringent asymmetric slotted core photonic crystal fiber in THz regime," IEEE Photon. Technol. Lett., vol. 27, no. 21, pp. 2222-2225, 2015.

[9] H.-B. Chen, D.-R. Chen, and Z. Hong, "Squeezed lattice elliptical-hole terahertz fiber with high birefringence," Appl. Opt., vol. 48, no. 20, pp. 3943-3947, 2009.

[10] N. Chen, J. Liangand, L. Ren, "High-birefringence, low-loss porous fiber for single-mode terahertz-wave guidance," Appl. Opt., vol. 52, no. 21, pp. 5297-5302, 2013.

[11] K. Nielsen, H. K. Rasmussen, A. J. Adam, P. C. Planken, O. Bang, and P. U. Jepsen, "Bendable, low-loss topas fibers for the terahertz frequency range," Opt. Exp., vol. 17, no. 10, pp. 8592-8601, May 2009.

[12] W. Yuan, L. Khan, D. J. Webb, K. Kalli, H. K. Rasmussen, A. Stefani, O. Bang, "Humidity insensitive TOPAS polymer fiber Bragg grating sensor," Opt. Express, vol. 19, no. 20, pp. 19731-19739, Sep. 2011.

[13] G. Emiliyanov, et al. "Localized biosensing with Topas microstructured polymer optical fiber," Opt. Lett, vol. 32, no. 5, pp. 460-62, 2007.

[14] A. W. Snyder and J. D. Love, Optical Waveguide Theory, London. U.K.: Chapman \& Hall, 1983.

[15] M. S. Habib, O. Bang, and M. Bache, "Low-loss hollow-core silica fibers with adjacent nested anti-resonant tubes," Opt. Express, vol. 23, no. 13, pp. 17394-17406, Jun. 2015.

[16] J. Liang, L. Ren, N, Chen, and C. Zhou, "Broadband, low-loss, dispersion flattened porous-core photonic bandgap fiber for terahertz (THz)-wave propagation," Opt. Commun., vol. 295, pp. 257-261, 2013.

[17] W. H. Reeves, J. C. Knight, and P. S. J. Russell, "Demonstration of ultra-flattened dispersion in photonic crystal fibers," Opt. Express, vol. 10, pp. 609-613, 2002.

[18] G. Barton, M. A. van Eijkelenborg, G. Henry, M. C. Large, and J. Zagari, "Fabrication of microstructured polymer optical fibres,"Opt. Fiber Technol., vol. 10, no.4, pp. 325-335, Oct. 2004.

[19] K. Nielsen, H. K. Rasmussen, P. U. Jepsen, and O. Bang, "Porous-core honeycomb bandgap THz fiber", Opt. Lett., vol. 36., No. 5, 2011. 